Theory \& Psychology, 2020, 31(1), 130-137

\title{
New materialism and postmodern subject models fail to explain human memory and self-awareness: A comment on Tobias-Renstrøm and Køppe (2020)
}

\author{
Radek Trnka \\ Prague College of Psychosocial Studies, Czech Republic \\ ORCID iD: https://orcid.org/0000-0003-3731-468X
}

\begin{abstract}
Tobias-Renstrøm and Køppe (2020) show the several conceptual limits that new materialism and postmodern subject models have for psychological theory and research. The present study continues in this discussion and argues that the applicability of the ideas of quantum-inspired new materialism depends on the theoretical perspectives that we consider for analysis: be it the first-person perspective referring to the subjective experience of a human subject, or the third-person perspective, in which a human subject is observed by an external observer. While the arguments of new materialism are in accordance with the analysis of the act of observation performed by an external observer, some problems arise when trying to theoretically approach the first-person subjective experience of a human subject. For example, new materialism fails to explain why human minds can maintain the awareness of a subject's identity throughout their lives and to recall the memories about their past personal experiences.
\end{abstract}

Keywords: subject models, quantum, observer effect, experience, consciousness, conscious experience, memory, self-awareness, theoretical psychology, new materialism, first-person perspective, third-person perspective, mind, body, sense of body ownership, identity, apparatus, observation, phenomenology, attention, mental states, mental processes, mental contents, past actions, past behaviors, past experiences, constant becoming, pre-existing relata, psyche, complexity, cognitive processing of past experiences

MeSH Headings: Consciousness, Awareness, Metacognition, Meta-cognition, Meta-cognitive Awareness, Metacognitive Awareness, Metacognitive Knowledge, Memory, Episodic Memory, Attention, Cognition

In line with Tobias-Renstrøm and Køppe (2020), this reflection aims to discuss some issues of postmodern subject models in terms of the theoretical analysis of the human psyche and consciousness. In a nutshell, postmodern subject models, particularly Barad's (2007) agential realism, propose that human subjects are suggested to be continually produced in the process of an intra-active becoming, all the time and everywhere. Within this perspective, human subjects are phenomena, and phenomena are suggested to exist without any prior existence of independent entities or relata. Entities are assumed to not pre-exist their intra-action, and before the so-called "agential cuts", entities are always indeterminate. Only by the force of intraactivity do the boundaries of subjects, meanings, and patterns become determinate in the ongoing flow of agential cuts, but only temporarily. The phenomena are ceaselessly being re-enfolded, reformed, and re-configurated. Moments of observation (or measurement) are examples of the "agential cuts". During agential cuts, the entities, e.g. human subjects, are assumed to emerge and are available to the senses or 
apparatuses of external observers. Any entity is produced through the complex agential intra-actions of multiple material-discursive practices or apparatuses. Prior to this intra-action, there is no such thing as an entity or human subject with distinguishable boundaries and identity. Simply put, entities become determinate repeatedly during the ongoing flow of agency. Barad (2007) speaks of an "ongoing reconfigurating of boundaries". The postmodern understanding of human subjects implies that human subjects are suggested to be constantly produced again and again in the flow of time. This approach does not consider the possibility of the continuous existence of entities and human subjects.

Tobias-Renstrøm and Køppe (2020) point out that this approach makes the investigation of the universal characteristics of human experiential life and any generalization of psychological findings impossible. This criticism argues against the assumption of postmodern subject models, where human bodies and human subjects are not objects with inherent boundaries and properties that pre-exist as such. The postmodern, new materialist's approach claims that knowledge, intentions, and subjectivity is not something that belongs to the human subject but is always a result of specific intra-actions. In contrast to this assumption, Tobias-Renstrøm and Køppe (2020) insist on the givenness of the human body which has universal characteristics. They also formulate the paradox of "multiple subjects relating to the same body". The postmodern, new materialist's assumption of the constant change and production of the subject enables the theoretical possibility of the emergence of multiple subjects relating to the same body (or even multiple bodies) in time flow. Simply put, if the boundaries and subjectivity of a human subject are not inherent and pre-exist each agential cut, then the identity of the human subject is not permanent and may vary across different moments in time.

This criticism is legitimate; however, every coin has two sides. The present reflection shows that the applicability of the quantum-inspired, new materialist subject models relies on a type of perspective that we shall further consider. Furthermore, we introduce new arguments showing that postmodern subject models also face other limitations, namely the impossibility of explaining human conscious self-awareness and storage and the later recollection of past personal experiences.

To begin with, two different perspectives of the theoretical analysis of a human subject have to be distinguished: a first-person perspective referring to a subjective experience involving a subject's own observation of its own internal milieu, and a thirdperson perspective, in which a human subject is observed by an external observer, e.g. a researcher (Pokropski, 2019; Trnka \& Smelik, 2020). First-person and thirdperson perspectives represent two different analytical positions. When considering human experience, the first-person perspective reflects the position of an "internal observer" and the process when a subject observes its own experiences, e.g. emotions, reasoning, proprioceptive sensing, or any other mental contents. In contrast, the third-person perspective reflects the position of an "external observer" and the process when one subject asks another subject about their actual or past internal experiences. If the external observer shifts his or her attention from another subject to their own experiences, the perspective changes and the third-person observation is changed to first-person observation.

The new materialist approach can be defended if we consider the position of an external observer that is observing a human subject. The idea of "agential cut" was inspired by the effect known in quantum mechanics as the "observer effect". Simply put, observer effect means that the act of observing will influence the appearance of a phenomenon (Trnka \& Lorencova, 2016). Let us take a closer look at this effect. There 
are three agencies that influence the emergence of the phenomena: the observer, the observed, and the apparatus used for the observation, e.g. technological devices commonly used in experimental and physiological psychology. The observed, the observer, and the apparatus interact together, and this interaction is understood as three intra-acting agencies that are present during the "agential cut", which play a constructive role in the appearance of phenomena (Barad, 2007; Huniche \& Sørensen, 2019). Various instances of observer effect are well-described also in psychology, where they are known as audience effect (Bateson et al., 2006; Cañigueral \& Hamilton, 2019), actor-observer bias (Jones \& Nisbett, 1972), the Hawthorne effect (Chiesa \& Hobbs, 2008), fundamental attribution error, or attribution bias (Ross, 2018; see also Burghardt et al., 2012).

When turning our attention to the first-person subjective experience of a human subject, the new materialist and postmodern understanding of human subjects faces several difficulties. As mentioned above, these approaches make the investigation of the universal characteristics of experiential life and the generalization of psychological findings impossible, because the subjectivity is not believed to be permanently tied to the human subject, and the boundaries of a human subject are suggested not to be determinate across time. However, these are not the only problems that the postmodern understanding of human subjects faces. There are at least two other, also fundamental problems: the problem of how to explain the continuous first-person access to a subject's experience of stream of consciousness and the process of recalling a subject's memories of the past.

Phenomenology, as well as empirical psychological research, has shown that people have first-person access to a continuous stream of their subjective experiences (Manning \& Manning, 2007; Trnka \& Smelik, 2020; Heavey \& Hurlburt, 2008). Empirical research has also shown that people remember their past life experiences and are able to recall at least some of these experiences from memory (Díaz, 2018; Rugg \& Vilberg, 2013). The concept of self-awareness denotes that the internal milieu of human subjects can become the object of a subject's own attention (Llinás, 2008; Morin, 2006). In other words, subjects can attend to their mental states, mental processes, and mental contents, also including an awareness of their identity, and can recall past actions, behaviors, and experiences.

Strikingly, these psychological findings prove to be difficult when interpreted from the position of new materialism and Barad's (2007) agential realism. If human subjects would be "made" again and again in the process of a constant, intra-active becoming without any pre-existing relata (Barad, 2007), one can seriously pose the question of how is it possible that human subjects have an awareness of their identity and their past experiences? If human subjects would not preexist their intra-action, how is possible that they have first-person access to their past actions, behaviors, and experiences? If human subjects would not have a distinguishable identity prior to a particular intra-action or agential cut, why are human subjects able to remember their identity and know "who they are" across different moments of time? Are self-awareness and memory retrieval only illusions or random artefacts of brain functioning? The current evidence of psychological research does not indicate so. Instead of assuming that human subjects constantly emerge during discrete moments of the flow of agential cuts, it seems that there is some continuity, at least in the subjective conscious experience of a subject's existence. The human ability to store past experiences in memory and to recall them later enables the maintenance of identity in a human subject. This ability enables the continuous experience of a subject's identity and the remembrance of a subject's personal characteristics in the time flow. It is necessary 
to say that not all past experiences of a subject are always stored in the subject's memory as fixed and invariable patterns. On the contrary, they are constantly modified by the cognitive processing of past experiences in the time flow, and some of them can be also lost in the process of forgetting (Conway \& Holmes, 2004). But they exist, at least subjectively and temporarily, and can be accessed through first-person introspection or even be communicated/shared with other human subjects in social interaction (Trnka \& Smelik, 2020; Morin, 2006).

Barad's (2007) agential realism and understanding of a constant, intra-active becoming was inspired by the theory of quantum mechanics. The idea of the quantum sea (Puthoff, 2002) assumes that a vacuum is never particle-free but consists of continuous processes of virtual particle-pair creation and annihilation. In the vacuum, these processes share some similarities with the idea of the constant, intra-active becoming proposed by the new materialist understanding of the human subject. Also, so-called zero-point fluctuations in the vacuum are random, fluctuating energies that are in a constant process of becoming. These fleeting electromagnetic waves and pairs of microparticles are assumed to perpetually come into being, only to return into nonexistence.

The effort to apply quantum principles to the theoretical understanding of the human subject as well as to the interpretation of human behavior and mental processes is certainly legitimate. Human bodies and brains are made of matter and energy (Trnka \& Lorencova, 2016). Matter and energy are basal substances from which all human subjects are built up. Quantum mechanics thus has great potential for informing and inspiring theoretical psychology and human subject models. However, the key question is how can the principles found in the research of microparticles be applied in theoretical psychology? Obviously, the answer is not straightforward. Quantum mechanics investigates objects that are certainly less complex systems than human beings are. Humans are much more complex systems than quantum microparticles, and as such, they also show some emergent properties, for example, memory and the conscious experience of the continuous persistence of their body and identity across time. Aside from some specific instances like amnesia, depersonalization disorder, or altered states of consciousness (Sierra \& David, 2011; Trnka, 2019), people are consciously aware of their identities, and this self-awareness is present even in moments when they are not observed by any external observer.

Past psychological research has revealed evidence for the continuous persistence of the relationship between a subject's body and a subject's conscious experience. The conscious experience of owning one's body (Martin, 1995) is an example of a first-person experience, when a subject is aware of their body over time. This coherent sense of body ownership (sense of embodiment) arises from the integration of interoceptive signals and external sensory inputs (Moseley et al., 2012; Tsakiris, 2010, 2017). This experience of embodiment is continuous and occurs automatically, without demand on cognitive effort (Fahey et. al, 2018).

Moreover, human subjects are also able to maintain a subjective sense of being the same individual and the same consciousness throughout their lives (Díaz, 2018). Thanks to the episodic memory system, the recording, acquisition, codification, and recollection of past personal experiences and their location in time is possible (Rugg \& Vilberg, 2013). Such memory repositories enable the creation of an autobiography and a narrative of a subject's own past.

The maintenance of a subjective sense of being by the same individual and subjective sense of body ownership throughout the lives of human subjects contrasts with the assumption of a chain of discontinuous moments of agential cuts, as supposed 
by new materialist subject models. As mentioned above, new materialism and postmodern subject models suppose that the human subject is produced during agential cuts without a prior existence of their distinguishable bodies and identities. The recent findings of psychological research do not indicate this. In contrast, psychological research shows that human subjects can keep a subjective sense of being the same individual and a subjective sense of body ownership throughout their lives. Under normal states of consciousness, the first-person access to a subject's autobiography, i.e. the narrative of a subject's own past, and body ownership does not seem to be dependent on agential intra-actions.

The aforementioned insights aim to stimulate future theory development in this field and to also formulate further fundamental questions regarding the nature of human subjects, the psyche, and the conscious experience of the world. Among other issues, future theoretical discussions should explore the embodied nature of selfawareness and the human ability to perceive and represent ourselves even at the prereflective level (Bielas \& Michalczyk, 2019; Ciaunica \& Crucianelli, 2019; NidaRümelin, 2017; Picolas \& Soueltzis, 2019). By positing the question of the unity of consciousness (Levin et al., 2019), how (and if) human subjects are able to integrate different components from the experience of streams of consciousness into one, integrative, superordinate internal stream of semiotic content should be explored (see e.g. Rafieian, 2012). Within the self-memory system model (Conway \& Holmes, 2004), it is also relevant to consider how the recollection and construction of past, self-related memories are shaped by personal goal-relevance. Future theoretical discussions should also explore if the conscious access to a subject's experience involves the selective routing of representational content to neural systems, and how such selective mechanisms could work (Smith, 2016).

\section{Author biography}

Radek Trnka is a lecturer and senior researcher at the Prague College of Psychosocial Studies and Palacky University Olomouc. His research interests include emotions, creativity, coping with stress, and the interactions between culture and the human mind. Within the philosophy of science scope, he aims to critically examine how the principles of quantum theory can inspire the newest trends in psychology and anthropology. He has contributed to the establishment of a new, arising crossdisciplinary field, "quantum anthropology," that seeks interconnections between quantum theoretical thinking, contemporary psychology, and anthropology. Most of the core issues of this framework were introduced in his recent book Quantum Anthropology: Man, Cultures, and Groups in a Quantum Perspective (Charles University Karolinum Press, 2016, available for download without cost here:

https://www.researchgate.net/publication/308792484_Quantum_Anthropology _Man_Cultures_and_Groups_in_a_Quantum_Perspective)

\section{References}

Barad, K. (2007). Meeting the Universe halfway: Quantum physics and entanglement of matter and meaning. Duke University Press. doi: 10.5334/opt.081013

Bateson, M., Nettle, D., \& Roberts, G. (2006). Cues of being watched enhance cooperation in a real-world setting. Biology Letters, 2(3), 412-414. doi: 10.1098/rsbl.2006.0509

Bielas, J., \& Michalczyk, Ł. (2019). Is the premotor theory of attention essentially about pre-reflective intentionality? Theory \& Psychology, 29(6), 757-774. doi: $10.1177 / 0959354319834672$ 
Burghardt, G. M., Bartmess-LeVasseur, J. N., Browning, S. A., Morrison, K. E., Stec, C. L., Zachau, C. E., \& Freeberg, T. M. (2012). Perspectives-minimizing observer bias in behavioral studies: A review and recommendations. Ethology, 118(6), 511-517. doi: 10.1111/j.14390310.2012.02040.x

Cañigueral, R., \& Hamilton, A. F. D. C. (2019). Effects of being watched on selfreferential processing, self-awareness and prosocial behaviour. Consciousness and Cognition, 76, 102830. doi: 10.1016/j.concog.2019.102830

Chiesa, M., \& Hobbs, S. (2008). Making sense of social research: How useful is the Hawthorne Effect? European Journal of Social Psychology, 38(1), 67-74. doi: 10.1002/ejsp.401

Ciaunica, A., \& Crucianelli, L. (2019). Minimal self-awareness: From within a developmental perspective. Journal of Consciousness Studies, 26(3-4), 207226.

Conway, M. A., \& Holmes, A. (2004). Psychosocial stages and the accessibility of autobiographical memories across the life cycle. Journal of Personality, 72(3), 461-480. doi: 10.1111/j.0022-3506.2004.00269.x

Díaz, J. L. (2018). Self-consciousness: An I-World patterned process model. Adaptive Behavior, 26(5), 211-223. doi: 10.1177/1059712318783434

Fahey, S., Charette, L., Francis, C., \& Zheng, Z. (2018). Multisensory integration of signals for bodily self-awareness requires minimal cognitive effort. Canadian Journal of Experimental Psychology/Revue Canadienne de Psychologie Expérimentale, 72(4), 244-252. doi: 10.1037/cep0000152

Heavey, C. L., \& Hurlburt, R. T. (2008). The phenomena of inner experience. Consciousness and Cognition, 17(3), 798-810. doi: 10.1016/j.concog.2007.12.006

Huniche, L., \& Sørensen, E. (2019). Phenomenon-driven research and systematic research assembling: Methodological conceptualisations for psychology's epistemic projects. Theory \& Psychology, 29(4), 539-558. doi: $10.1177 / 0959354319862048$

Jones, E. E., \& Nisbett, R. E. (1972). The actor and the observer: Divergent perceptions in the causes of behavior. In E. E. Jones, D. E. Kanouse, H. H. Kelley, R. E. Nisbett, S. Valins, \& B. Weiner (Eds.), Attribution: Perceiving the causes of behavior (pp. 79-94). General Learning.

Levin, K., Køppe, S., \& Roald, T. (2019). Modalization of movement: The problem of sensus communis and the limits of phenomenology. Theory \& Psychology, 29(6), 833-852. doi: 10.1177/0959354319888685

Llinás, R. (2008). Of self and self awareness: The basic neuronal circuit in human consciousness and the generation of self. Journal of Consciousness Studies, 15(9), 64-74.

Manning, M. L., \& Manning, R. L. (2007). Legion theory: A meta-psychology. Theory \& Psychology, 17(6), 839-862. doi: 10.1177/0959354307083497

Martin, M. G. F. (1995). Bodily awareness: A sense of ownership. In J.L. Bermudez, A. Marcel, \& N. Elian (Eds.), The body and the self (pp. 267-289). MIT Press.

Morin, A. (2006). Levels of consciousness and self-awareness: A comparison and integration of various neurocognitive views. Consciousness and Cognition, 15(2), 358-371. doi: 10.1016/j.concog.2005.09.006

Moseley, G. L., Gallace, A., \& Spence, C. (2012). Bodily illusions in health and disease: Physiological and clinical perspectives and the concept of a cortical 
'body matrix'. Neuroscience \& Biobehavioral Reviews, 36(1), 34-46. doi: 10.1016/j.neubiorev.2011.03.013

Nida-Rümelin, M. (2017). Self-awareness. Review of Philosophy and Psychology, 8(1), 55-82. doi: 10.1007/s13164-016-0328-x

Picolas, C., \& Soueltzis, N. (2019). Bodily and temporal pre-reflective selfawareness. Phenomenology and the Cognitive Sciences, 18(3), 603-620. doi: $10.1007 / \mathrm{s} 11097-018-9589-4$

Pokropski, M. (2019). Phenomenology and mechanisms of consciousness: Considering the theoretical integration of phenomenology with a mechanistic framework. Theory \& Psychology, 29(5), 601-619. doi: $10.1177 / 0959354319868769$

Puthoff, H. E. (2002). Searching for the universal matrix in metaphysics. Research News and Opportunities in Science and Theology, 2(8), 22-24.

Rafieian, S. (2012). A biosemiotic approach to the problem of structure and agency. Biosemiotics, 5(1), 83-93. doi: 10.1007/s12304-011-9121-5

Ross, L. (2018). From the fundamental attribution error to the truly fundamental attribution error and beyond: My research journey. Perspectives on Psychological Science, 13(6), 750-769. doi: 10.1177/1745691618769855

Rugg, M. D., \& Vilberg, K. L. (2013). Brain networks underlying episodic memory retrieval. Current Opinion in Neurobiology, 23(2), 255-260. doi: 10.1016/j.conb.2012.11.005

Sierra, M., \& David, A. S. (2011). Depersonalization: A selective impairment of selfawareness. Consciousness and Cognition, 20(1), 99-108. doi: 10.1016/j.concog.2010.10.018

Smith, R. (2016). The relationship between consciousness, understanding, and rationality. Philosophical Psychology, 29(7), 943-957. doi: 10.1080/09515089.2016.1172700

Tobias-Renstrøm, S., \& Køppe, S. (2020). Karen Barad, psychology, and subject models: Why we need to take experience seriously. Theory \& Psychology. doi: 10.1177/0959354320903089

Trnka (2019). Human beings in quantum anthropology: A paradox of the discontinuous experience of quantum spacetime. [Invited lecture]. Department of Anthropology, University of Copenhagen, Denmark.

https://www.researchgate.net/publication/332442510_Human_Beings_in_Qua ntum_Anthropology_A_Paradox_of_the_Discontinuous_Experience_of_Quant um_Spacetime

Trnka, R., \& Lorencova, R. (2016). Quantum anthropology: Man, cultures, and groups in a quantum perspective. Charles University Karolinum Press. doi: 10.13140/RG.2.2.20009.80485

https://www.researchgate.net/publication/308792484_Quantum_Anthropology Man_Cultures_and_Groups_in_a_Quantum_Perspective

Trnka, $\bar{R} ., \&$ Smelik, V. (2020). Elimination of bias in introspection: Methodological advances, refinements, and recommendations. New ldeas in Psychology, 56, doi: 10.1016/j.newideapsych.2019.100753

Tsakiris, M. (2010). My body in the brain: A neurocognitive model of bodyownership. Neuropsychologia, 48(3), 703-712. doi: 10.1016/j.neuropsychologia.2009.09.034

Tsakiris, M. (2017). The multisensory basis of the self: From body to identity to others. The Quarterly Journal of Experimental Psychology, 70(4), 597-609. doi: 10.1080/17470218.2016.1181768 